\title{
1 Subsurface microbial habitats in an extreme desert Mars-analogue
}

\section{2 environment}

4 Kimberley A. Warren-Rhodes ${ }^{1,2}$, Kevin C. Lee ${ }^{3}$, Stephen D.J. Archer ${ }^{3}$, Nathalie Cabrol ${ }^{1,2}$, Linda

$5 \quad$ Ng-Boyle ${ }^{4}$, David Wettergreen ${ }^{5}$, Kris Zacny ${ }^{6}$, Stephen B. Pointing ${ }^{7 *}$ and the NASA Life in the

6 Atacama Project Team

$8 \quad{ }^{1}$ NASA Ames Research Center, Moffett Field, CA 94035, USA

$9 \quad 2$ The SETI Institute, Mountain View, CA 94043, USA

$10{ }^{3}$ School of Sciences, Auckland University of Technology, Auckland 1142, New Zealand

$11{ }^{4}$ College of Engineering, University of Washington, Seattle, WA 98195, USA

$12{ }^{5}$ Institute of Robotics, Carnegie-Mellon University, Pittsburgh, PA 15213, USA

$13{ }^{6}$ Honeybee Robotics and Spacecraft Mechanisms Corp., Pasadena, CA 91103, USA

$14{ }^{7}$ Yale-NUS College and Department of Biological Sciences, National University of Singapore,

15 Singapore 138527

$16 \quad$ *Corresponding author: Email: stephen.pointing@yale-nus.edu.sg

18 Abstract

19 Sediments in the hyper-arid core of the Atacama Desert are a terrestrial analogue to Mars regolith.

20 Understanding the distribution and drivers of microbial life in the sediment may give critical clues

21 on how to search for biosignatures on Mars. Here, we identify the spatial distribution of highly

22 specialised bacterial communities in previously unexplored depth horizons of subsurface

23 sediments. We deployed an autonomous rover in a mission-relevant Martian drilling scenario with

24 manual sample validation. Subsurface communities were delineated by depth related to sediment 
1 moisture. Geochemical analysis indicated soluble salts and minerology that influenced water bio-

2 availability, particularly in deeper sediments. Colonization was also patchy and uncolonized

3 sediment was associated with indicators of extreme osmotic challenge. The study identifies

4 linkage between biocomplexity, moisture and geochemistry in Mars-like sediments at the limit of

5 habitability and demonstrates feasibility of the rover-mounted drill for future Mars sample

6 recovery.

$8 \quad$ Keywords

9 Atacama; Desert soil; Mars, Moisture stress, Soil bacteria

11 Introduction

12 The surface of Mars is dry, cold, and exposed to high levels of ionizing radiation. However, data

13 accumulated over the past few decades by orbital and landed missions have demonstrated that

14 early in its history the planet may have been habitable for microbial life with abundant sources of

15 energy, carbon, nutrients, and shelter (Cabrol, 2018). Mars supported surface and subsurface

16 water and may still do in some circumstances, as well as organic molecules required for life

17 (Martin-Torres et al., 2015). As a result, Mars 2020 and ExoMars missions will be searching for

18 biosignatures (Farley and Williford, 2017; Vago et al., 2017), and the investigation of terrestrial

19 analogues can provide critical insights for the development and testing of exploration strategies.

20 Among those, the hyper-arid core of the Atacama Desert in Chile is widely regarded as a tractable

21 Mars analogue in the field of astrobiology. The Atacama is the driest desert region on Earth (Peel

22 and Finlayson, 2007) with extremely low moisture inputs and precipitation events that are

23 stochastic in nature (McKay et al., 2003). The region has a long history of climatic stability as an

24 extreme desert (Clarke, 2006; Hartley et al., 2005) resulting in the build-up of evaporates (McKay 
1 et al., 2003; Navarro-Gonzalez et al., 2003) and creation of a Mars-like surface. The desert's

2 hyper-arid core lies at or near the arid limit for soil formation (Ewing et al., 2006) and thus

3 surface terrain in this region is regarded as sediment.

Animal and plant life are scarce in extreme deserts and instead cyanobacteria-dominated

5 microbial communities in mineral and rocky refugia including deliquescent substrates comprise

6 the dominant surface biota and are well-characterized (Azua-Bustos et al., 2012; Pointing and

7 Belnap, 2012). Conversely, evidence for microbial colonization in hyper-arid Atacama sediment

8 is scarce, contradictory and almost exclusively limited to surface-associated sediment (Connon et

9 al., 2007; Crits-Christoph et al., 2013; Drees et al., 2006; Lester et al., 2007; Navarro-Gonzalez et

10 al., 2003; Schulze-Makuch et al., 2018) (Supplementary Material, Table S1). Cultivation-based

11 approaches are unreliable as indicators of environmental microbial diversity and have yielded

12 estimates that varied by several orders of magnitude (Bagaley, 2006; Connon et al., 2007; Lester

13 et al., 2007; Navarro-Gonzalez et al., 2003; Schulze-Makuch et al., 2018). Biochemical tests have

14 similarly yielded inconclusive support for microbial metabolic activity (Connon et al., 2007;

15 Crits-Christoph et al., 2013; Lester et al., 2007; Navarro-Gonzalez et al., 2003; Schulze-Makuch

16 et al., 2018). High throughput sequencing of environmental DNA/RNA remains the most reliable

17 indicator for microbial diversity in extreme desert sediments. This approach has provided critical

18 insight on surface-associated communities in semi-arid, arid and hyper-arid locations (Crits-

19 Christoph et al., 2013). A recent study also estimated putative microbial metabolic activity in

20 three subsurface samples after an unusual rain event and this suggested subsurface sediment may

21 also be a habitat for microbial communities (Schulze-Makuch et al., 2018). Major knowledge

22 gaps persist, and importantly from an astrobiology perspective the question of how microbial

23 occurrence and diversity may vary with abiotic variables in sediment depth horizons of a Mars

24 analogue and spatially within terrain, and whether this can be addressed in a simulated robotic

25 sampling mission scenario. 
To document these questions and support the development and testing of biosignature

exploration strategies, the NASA-funded Subsurface Life in the Atacama (LITA) project

deployed an autonomous rover-mounted robotic drill in Mars-like desert pavement and playa

terrain within the hyper-arid core of the Atacama under challenging environmental and logistical

constraints (Supplementary Material, Fig. S1-S3). The drill accessed 32 discreet sediment

samples to depths of $800 \mathrm{~mm}$ along a $50 \mathrm{~km}$ transect in a realistic simulation of Martian drilling

operations and constraints. Over 60 manually excavated sediment samples were recovered in

parallel and validated the automated sampling for abiotic and biotic components of the sediment

habitat and provide important baseline ecological data on the most Mars-like sediments on Earth (Warren-Rhodes et al., 2018).

\section{Materials and Methods}

\section{Field sites}

A 50-km autonomous rover traverse in the hyper-arid core of the Atacama Desert was completed in 2013 under challenging environmental and logistical conditions (Supplementary Material Fig. S1). The field experiment took place over a period of two weeks within a landing ellipse circumscribed by rover logistical constraints, ASTER satellite geochemistry and historical climate data as appropriate Mars analogues. The rover traversed two types of terrain identified as appropriate Mars analogues (McKay et al., 2003): stony desert pavement in the western part of the traverse and desert playa in the eastern part within the topographical low and terminus for water runoff (snowmelt, rainfall) for the surrounding region (thus likely to receive significantly more moisture from easterly winter Andean precipitation and runoff than the desert pavement). A unique paleo-playa was also encountered and sampled, comprising an elevated area of dry sediment with evidence of historical playa features. A total of 61 manual samples and 32 roveracquired samples were independently collected and interrogated. 


\section{Robotic sample recovery}

2 The Zöe rover built by the Robotics Institute at Carnegie Mellon University is a solar-powered

3 rover designed to autonomously map and analyze contextual landscape and habitat visible and

4 geochemical features (with on-board navigation cameras and Vis-NIR spectrometer on its mast)

5 and to drill and deliver samples to on-board scientific instrumentation, including a Mars Micro-

6 beam Raman Spectrometer (MMRS) (Supplementary Material, Fig. S2). The drill, developed by

7 Honeybee Robotics Corporation is a $15 \mathrm{~kg}, 300-$ Watt, rotary-percussive and fully autonomous

8 drill designed to capture powdered rocks and sediment samples (Online Supplementary Material

9 Fig. S3). The drill consists of a rotary-percussive drill head, sampling augur, brushing station,

10 feed stage and deployment stage, using a vertical $19.1 \mathrm{~mm}$ diameter drill operating at $120 \mathrm{rpm}$.

11 Drilling is accomplished using a bite-sampling approach where samples are captured in 10-20 cm

12 intervals, to simulate a Martian drilling scenario (Zacny et al., 2014). That is, after drilling 10-20

$13 \mathrm{~cm}$, the auger bit with the sample is pulled out of the hole, and the sample brushed off into an on-

14 board sample carousel cup (or sterile Whirlpak (Nasco) bag for manual recovery). Initially

15 autoclaved in the laboratory, the drill bit, brushing station, Z-stage and deployment stage were

16 field sterilized with $70 \%$ ethanol prior to and after each site sampling. Aseptic techniques were

17 also used throughout rover sampling operations, including minimal disturbance near the rover

18 during all collections.

\section{Manual Sediment Pit Sampling}

20 Post-drill, a sediment pit adjacent to the drill hole was excavated manually after first sampling

21 surface sediments. Samples were recovered horizontally from the exposed sediment horizon at 10

$22 \mathrm{~cm}$ depth intervals. The pit wall was excavated using a plastic Sterileware (Bel-Art Products)

23 scoop or metal trowel sterilized with $70 \%$ ethanol. Deeper layers occasionally required a drill to

24 obtain sample, and a Makita LXT drill was employed, with the drill bit first sterilized with $70 \%$

25 ethanol. All samples were collected using aseptic techniques and tools using a Sterileware (Bel- 
1 Art Products) sampling spatula or a stainless-steel spatula sterilized with $70 \%$ ethanol. Sediment

samples (50-200 g) were collected from each depth layer for biology and geochemistry, with care taken to minimize mixing between different depths, and placed immediately into sterile falcon tubes or Whirlpak (Nasco) bags. Samples were stored at $-80^{\circ} \mathrm{C}$ until processed in the laboratory.

\section{Mineralogical analysis using rover payload}

The MMRS on board the rover collected real-time in-situ mineralogical/geochemical analyses of sediment samples. This allowed direct and unambiguous molecular phase identification from raw Raman spectra of mixtures, e.g., minerals and sediments. The MMRS had a two-unit configuration connected using fiber-optics: A probe unit contained a YAG-KTP crystal pair to generate a $532 \mathrm{~nm}$ laser beam for excitation, the optics for laser beam focusing and for Raman photon collection, and a step-motoring mechanism that enable a $\sim 1 \mathrm{~cm}$ line scan at the sample surface. The main unit of the MMRS contained an $808 \mathrm{~nm}$ diode pump laser, MMRS Raman spectrometer and detector, electronics for laser driving and control, a microprocessor for commending MMRS operation and data receiving, plus MMRS calibration lamp. This covered a spectral range from $100-4000 \mathrm{~cm}^{-1}$ with a spectral resolution of $7-8 \mathrm{~cm}^{-1}$. The MMRS probe had a working distance of $\sim 10 \mathrm{~mm}$ from the sample surface and generated a laser beam of $20-30 \mathrm{~mW}$ with a diameter $<20 \mu \mathrm{m}$ at the focus. The wavelength calibration of MMRS spectrometer was performed twice using the Ne calibration lamp during the field campaign, and the laser wavelength calibration was performed daily using naphthalene powder. Raman spectra acquired by the rover were validated against laboratory analysis of sediments using a Kaiser Hololab 5000 — $532 \mathrm{~nm}$ Raman spectrometer with similar configuration to the MMRS and comparable performances in optical throughput (Wei et al., 2015).

\section{Sediment geochemistry and microclimate data}

In the laboratory, sediment chemical analyses for variables known to affect microbial colonization of soil/sediments, including $\mathrm{pH}$, electrical conductivity, soluble salts, $\mathrm{C}, \mathrm{N}, \mathrm{C}: \mathrm{N}$ ratio, extractable 
cations, phosphorous and sulfur plus inherent sediment properties including anion and cation exchange capacity and bulk density were measured according to standard soil chemical analysis methods (https://www.landcareresearch.co.nz/resources/laboratories/environmental-chemistrylaboratory/services/soil-testing/methods). It was recognized that high salinity and gypsum levels encountered in our desert sediments may influence estimates for water-soluble ions, however by adopting standard methods applied to soil testing in microbial ecology studies of soil globally we produced a data set that was comparable with other microbial diversity studies.

In brief, geochemical tests were conducted using the following methodology: All sediments were dried in a forced air convection drier at $35^{\circ} \mathrm{C}$, and after drying, sediments were crushed to pass through a $2 \mathrm{~mm}$ sieve. For $\mathrm{pH}, 10 \mathrm{~mL}$ of sediment was slurried with $20 \mathrm{~mL}$ of water, and after standing, the $\mathrm{pH}$ was measured (1:2 v/v slurry). Bulk density was obtained with a fixed volume $(12 \mathrm{~mL})$ of dried and ground sediment weighed. Phosphorus was extracted using Olsen's procedure (0.5M sodium bicarbonate, $\mathrm{pH} 8.5,1: 20 \mathrm{v} / \mathrm{v}$ sediment:extractant ratio, 30 minutes extraction), and the extracted phosphate was determined calorimetrically by a molybdenum blue procedure. Cations $\left(\mathrm{K}^{+}, \mathrm{Ca}^{2+}, \mathrm{Mg}^{2+}, \mathrm{Na}^{+}\right)$were extracted using ammonium acetate (1.0M, pH 7, 1:20 v/v sediment:extractant ratio, 30 minutes extraction), and determined by ICP-OES. Cation Exchange Capacity (CEC) was calculated by summation of the extractable cations and the extractable acidity. The extractable acidity was determined from the decrease in $\mathrm{pH}$ of the buffered ammonium acetate cation extract. For sulfate-sulfur, sediments were extracted using 0.02M potassium dihydrogen phosphate after 30 minutes shaking, and sulfate-sulfur was measured by anion-exchange chromatography (IC). Total nitrogen (TN) and total carbon (TC) were determined by the Dumas method of combustion. Each sample was combusted to produce varying proportions of $\mathrm{CH}_{4}$ and $\mathrm{CO}$ gas. The $\mathrm{CH}_{4}$ and $\mathrm{CO}$ gas was oxidized to $\mathrm{CO}_{2}$ using the catalysts Copper Oxide and Platinum. The $\mathrm{CO}_{2}$ was measured using Thermal Conductivity detector. Available nitrogen and anaerobically mineralizable nitrogen (not reported) were 
1 estimated after incubating sediment samples for seven days at $40^{\circ} \mathrm{C}$, after which the ammonium-

$2 \mathrm{~N}$ was extracted with potassium chloride (2M potassium chloride, 1:5 v/v sediment:extractant

3 ratio, 15 minutes shaking), and determined calorimetrically. A water extraction (1:5 w/v

4 sediment:extractant ratio, 30minutes shaking) was used for electrical conductivity, (EC), which

5 has be used as a proxy for water availability in desert soils/sediments, i.e., higher EC $=$ more salt,

6 less water (Crits-Christoph et al., 2013).

Micro-climate data were collected using in situ data loggers in pits installed at the date of sediment sampling in 2013 and recorded continuous subsurface microclimate for 14 months.

9 Relative humidity/temperature HOBO Pro v2 dataloggers (U23-002) were placed at $10 \mathrm{~cm}, 30 \mathrm{~cm}$ 10 and $80 \mathrm{~cm}$ depth in pavement and playa sites. Sediment water content was estimated

11 gravimetrically, directly from sediment samples in the laboratory after drying at $120^{\circ} \mathrm{C} \mathrm{x} 48 \mathrm{hrs}$ to 12 constant dry weight.

\section{Environmental 16S rRNA gene-defined diversity}

Total environmental genomic DNA were extracted from the sediment samples using a method proven during our previous research to maximize recovery from microbial communities from extreme environments. Sediment samples $(3 \mathrm{x} 0.5 \mathrm{~g}$ triplicate extractions per sample) were combined with $0.5 \mathrm{~g}$ of $0.1 \mathrm{~mm}$ silica-zirconia beads. To each sample, $320 \mu \mathrm{L}$ of phosphate buffer $\left(100 \mathrm{mM} \mathrm{NaH}_{2} \mathrm{PO}_{4}\right)$ and $320 \mu \mathrm{L}$ of SDS lysis buffer $(100 \mathrm{Mm} \mathrm{NaCl}, 500 \mathrm{mM}$ Tris $\mathrm{pH} 8.0,10 \%$

SDS) were added and samples were homogenized using the FastPrep®-24 (MP Biomedicals, Ohio, USA) at 5.5 for a 30 second run. Samples were centrifuged at 13,200 rpm for 3 minutes $\left(20^{\circ} \mathrm{C}\right)$ and $230 \mu \mathrm{L}$ of cetyltrimethylammonium bromide-polyvinylpyrrolidone (CTAB) extraction buffer (100 Mm Tis-HCl, 1.4 M NaCl, $20 \mathrm{Mm}$ EDTA, 2\% CTAB, 1\% polyvinylpyrrolidone and $0.4 \% \beta$-mecaptoethanol) was added. Samples were vortexed for $10 \mathrm{~s}$ before incubation at $60^{\circ} \mathrm{C}$ and $300 \mathrm{rpm}$ for $30 \mathrm{~min}$. Samples were centrifuged at 13,200 rpm for $1 \mathrm{~min}$ and then $400 \mu \mathrm{L}$ of chloroform:isoamyl alcohol (24:1) was added. Samples were then vortexed for $10 \mathrm{~s}$ and 
centrifuged at 13,200 rpm for $5 \mathrm{~min}$. The upper aqueous layer was removed into a new Eppendorf tube and a further $550 \mu \mathrm{L}$ of chloroform:isoamyl alcohol (24:1) was added. Samples were vortexed for $15 \mathrm{~s}$ and again centrifuged at 13,200 rpm for $5 \mathrm{~min}$. The upper aqueous phase was removed into a new Eppendorf tube and $10 \mathrm{M}$ ammonium acetate was added to samples to achieve a final concentration of 2.5 M. Samples were vortexed for $10 \mathrm{~s}$ and centrifuged at 13,200 rpm for $5 \mathrm{~min}$. The aqueous layer was removed to a new tube and 0.54 volume of isopropanol was added and mixed by inversion. Samples were left for 24 hours at $-20^{\circ} \mathrm{C}$ and then centrifuged for 20 minutes at $13,200 \mathrm{rpm}\left(4^{\circ} \mathrm{C}\right)$. The supernatant was discarded and the pellet was washed with $1 \mathrm{~mL} \mathrm{70 \%} \mathrm{ethanol} \mathrm{and} \mathrm{centrifuged} \mathrm{for} 10 \mathrm{~min}$ at 13,200 rpm. Ethanol was removed and DNA was re-suspended in $20 \mu \mathrm{L}$ of sterile DNase free water. Samples were then quantified using the Qubit 2.0 Flourometer (Invitrogen). Samples were then stored at $-20^{\circ} \mathrm{C}$ until required. The extracted DNA were then adjusted, where possible, to $5 \mathrm{ng} / \mu \mathrm{L}$ before Illumina MiSeq library preparation as specified by the manufacturer (16S Metagenomic Sequencing Library Preparation Part \# 15044223 Rev. B; Illumina, San Diego, CA, USA). Briefly, PCR was conducted with the primer set targeting the V3-V4 regions of bacterial and archaeal 16S rRNA gene: PCR1 forward (5' TCGTCGGCAG CGTCAGATGT GTATAAGAGA CAGCCTACGG GNGGCWGCAG 3') and PCR1 reverse (5' GTCTCGTGGG CTCGGAGATG TGTATAAGAG ACAGGACTAC HVGGGTATCT AATCC 3') with KAPA HiFi Hotstart Readymix (Kapa Biosystems, Wilmington, MA, USA) and the following thermocycling parameters: (1) $95^{\circ} \mathrm{C}$ for $3 \mathrm{~min}$, (2) 25 cycles of $95^{\circ} \mathrm{C}$ for $30 \mathrm{~s}, 55^{\circ} \mathrm{C}$ for $30 \mathrm{~s}, 72^{\circ} \mathrm{C}$ for $30 \mathrm{~s}, 72^{\circ} \mathrm{C}$ for $5 \mathrm{~min}$, and (3) holding the samples at $4{ }^{\circ} \mathrm{C}$. The amplicons were then indexed using Nextera XT index kit (Illumina). The indexed amplicons were purified and size selected using AMPure XP beads (Beckman-Coulter, Brea, CA, USA) before sequencing on an Illumina Miseq (Illumina) with the 500 cycle V2 chemistry (250 bp paired-end reads). A 5\% PhiX spike-in was used, as per manufacturer's recommendation. The $\mathrm{R}$ packages phyloseq, DESeq2 and ggplot2 were used for downstream analysis and visualization 
1 including ordination and alpha diversity calculations (https://www.r-project.org/). High-

2 throughput sequencing of the 16S rRNA gene yielded 87,8875 quality filtered reads and 92

3 bacterial OTUs that were further analyzed. All sequence data acquired during this investigation

4 has been deposited in the NCBI Sequence Read Archive under project accession number

5 PRJEB22902.

Our approach employed an environmental DNA recovery method that has been optimized

7 for extreme desert sediments and has been used as a proxy for relative biomass(Pointing et al.,

8 2009). We employed positive and negative controls for all PCR amplifications. We employed

9 rRNA gene loci widely accepted as the benchmark for interrogating environmental soil/sediment

10 samples (http://www.earthmicrobiome.org/). We also adopted high coverage and carefully

11 screened our sequence libraries for artefacts and contaminants. We also performed successful

12 DNA extractions on pavement and playa sediments that yielded negative extraction outcomes for

13 environmental DNA (as well as those yielding positive outcomes as controls), after spiking these

14 with E.coli from an axenic cell suspension in phosphate buffered saline solution at final cell

15 concentrations of $10^{3}-10^{7} \mathrm{cfu} / \mathrm{g}$ sediment to simulate estimated occurrence in hyper arid

16 sediment (Connon et al., 2007; Lester et al., 2007). Recovery rates were in the range of $60-80 \%$ of

17 that for axenic cell suspensions in all treatments.

We did not supplement our intensive molecular ecological survey with a cultivation

19 approach because it is widely accepted that most environmental bacteria are unculturable and

20 their in vitro growth requirements are unknown, thus such an approach would have introduced

21 unacceptable bias to the study. Similarly, the lack of resolution and triangulation available for

22 some alternative approaches including lipid analysis and metabolic activity limit their

23 contribution to microbial community estimation relative to DNA sequencing approaches. Given

24 the inherent patchiness of distribution for microbial life in extreme deserts and our robust 
1 methodological approach we are confident that our estimates provided the most accurate possible

2 indication of endemic diversity.

$3 \quad$ Statistical analysis

4 Canonical correspondence analysis (CCA) was performed with the $\mathrm{R}$ package vegan to explore

5 the strength of associations among the sediment geochemistry profiles, bacterial taxa (OTUs) and

6 site locations (https://www.r-project.org/). Type III symmetrical scaling was used in the CCA

7 plot, where both site and species scores were scaled symmetrically by square root of eigenvalues.

8 This technique provided a weighted sum of the variables that maximizes the correlation between

9 the canonical variates. A biplot was created to visualize the outcomes and help facilitate

10 interpretation of the canonical variate scores. Linear Discriminant Analysis (LDA) using the R

11 package flipMultivariates (https://github.com/Displayr/flipMultivariates) was applied to

12 geochemistry data (Online Supplementary Table S3) (n=61, cases used for estimation $n=47$,

13 values below detection range were treated as 0 , cases containing missing values were excluded,

14 null hypotheses $=$ two-sided). For multiple comparisons correction, False Discovery Rate

15 correction was applied simultaneously to the entire table. BEST analyses were conducted using

16 the BIO-ENV procedure in Primer 7 software (http://www.primer-e.com/) to maximize the rank

17 correlation between biotic and environmental data, thereby establishing a ranking $(p w)$ for the

18 effects of environmental variables on diversity.

$20 \quad$ Results and Discussion

21 Extreme habitats in Atacama sediment horizons

22 The on-board Raman spectrometry revealed surface mineral distributions comprising feldspar and

23 quartz. Subsurface sediments, and particularly the playa sites, were elevated in gypsum and

24 anhydrite (Supplementary Material, Table S3, Fig. S5). This was independently corroborated by

25 laboratory Raman measurements (Supplementary Material, Table S3) and geochemical analysis 
1 that revealed a positive linear correlation $(\mathrm{r}=0.78, \mathrm{p}<0.001)$ across all samples between sulfate-

2 sulfur and calcium, the components of gypsum and anhydrite $\left(\mathrm{CaSO}_{4}+/-2 \mathrm{H}_{2} \mathrm{O}\right)$. Anydrite is

3 indicative of drier conditions at depth, and in situ soil moisture sensors validated this for horizons

$4 \quad$ in both pavement and playa.

5 Our chemical analysis focused on geochemical reservoirs readily available and relevant to

6 biological communities and using methodology that was comparable with other microbial ecology

7 studies. A clear depth-dependent pattern in geochemistry was revealed for pavement and playa

8 horizons (Fig. 1, Supplementary Material, Table S2, Fig. S4). Surface sediments were strongly

9 associated with elevated phosphorous concentrations in both playa and pavement units (Fig. 1).

10 Elevated surface $\mathrm{P}$ was attributed to presence of surface Fe-oxides and calcite that can adsorb P.

11 These P-adsorbing phases were likely present in the subsurface but were diluted by high sulfate

12 concentrations causing lower recorded $\mathrm{P}$ concentrations below the surface. All subsurface

13 samples displayed very low to undetectable levels of $\mathrm{N}$ and low $\mathrm{C}$ throughout horizons. The on-

14 board Raman spectrometer also recorded $\mathrm{C}$ as a minor/undetectable phase in all samples.

15 Electrical conductivity (EC) and $\mathrm{Na}^{+}$levels increased with depth in both substrates indicating

16 increased salinity with depth $(\mathrm{p}=0.006)$. Desert pavement subsurface sediments separated mainly

17 due to $\mathrm{pH}$ and $\mathrm{K}^{+}$, whilst playa subsurface sediments displayed elevated levels of sulfate-sulfur,

18 extractable cations $\left(\mathrm{Ca}^{2+}, \mathrm{Mg}^{2+}, \mathrm{Na}^{+}\right)$and EC indicating increasing osmotic challenge, and most

19 notably in the paleo-playa Site 10 (Fig. 1, Supplementary Material Table S2).

Average annual subsurface temperatures ranged from $19.9-20.9^{\circ} \mathrm{C}$ in depth horizons

21 (Supplementary Material, Table S4). At shallow depths $(<100 \mathrm{~mm})$ temperature varied between 4

$22-34^{\circ} \mathrm{C}$, whereas in deeper horizons temperature variation was somewhat buffered

23 (Supplementary Material, Fig. S6). Moisture values from depth horizons in representative playa

24 terrain were consistently higher (approximately 4- to 7-fold) at all depths than those in the desert

25 pavement horizons (Supplementary Material, Table S4) and this likely reflects local hydrology 
1 given the playa was a relatively low-lying terrain (Supplementary Material, Fig. S1). However,

2 sediment moisture trends in both substrates broadly indicated the existence of depth groupings

3 into distinct moisture zones: (a) a surface zone consisting of the top 200mm, where water

4 availability is typically lowest, except in the short-term following a rain event; (b) a mid-depth

5 zone (300-500mm), where water availability peaks and persists after a rain event; and (c) a deep

6 subsurface zone ( $\geq 500-800 \mathrm{~mm})$, where water availability is typically lower and, notably for the

7 desert pavement sediments, appeared to be un-impacted by rare large rainfall events

8 (Supplementary Material, Table S4, Fig. S6).

10 Depth-related trends in DNA recovery and microbial alpha diversity

11 Surface colonization was widespread in desert pavement and playa sites but subsurface horizons

12 displayed patchy recovery with low yields of quantifiable DNA in the range 0.067-6.5 ng/g

13 sediment, indicating extremely low standing biomass (Supplementary Material, Table S2, S5).

14 Notably, the paleo-playa site yielded no recoverable DNA at any depth after repeated efforts at

15 extraction. Our approach using a DNA recovery method adapted for low-biomass extreme

16 environments highlights for the first time the patchiness of low biomass microbial colonization in

17 the most extreme subsurface desert sediments where micro-habitat conditions are at or near the

18 limit for life (McKay, 2014). This may explain, at least in part, why some previous research has

19 concluded this region of the Atacama was lifeless (Navarro-Gonzalez et al., 2003). Linear

20 Discriminant Analysis was employed to show that variables most strongly associated with

21 potentially lifeless subsurface sediments where environmental DNA was irrecoverable were

22 sulfate-sulfur (substrate) $(\mathrm{p}=0.001)$, depth $(\mathrm{p}=0.003), \mathrm{EC}(\mathrm{p}=0.006)$, soluble salts $(\mathrm{p}=0.006)$.

23 This strongly suggests that osmotic challenge and limited moisture availability are the major

24 extinction drivers in this Mars analogue sediment. We also demonstrated that substrate-inhibition

25 of DNA recovery was unlikely to have been a significant factor, since bacterial cell suspensions 
1 in the range reported for desert soils (Supplementary Material, Table S1) added to these samples

2 were recoverable with 60-80\% efficiency compared to a pure laboratory culture and were not

3 significantly inhibited compared to extractions with spiked sediments that did originally yield

4 environmental DNA.

5 Across both terrains any given depth horizon tended to either be colonized almost

6 throughout or display only patchy near-surface colonization as measured by recoverable DNA.

7 Thus, the ubiquitous surface colonization was not a predictor for subsurface habitability in these

8 sediments. We postulate that in addition to sediment moisture, extreme geochemistry also

9 influenced habitability in these sediments as evidenced by high soluble salts/salinity indicators

10 and abundance of anhydrite and gypsum mineral signatures (Supplementary Material, Table S2,

$11 \mathrm{~S} 3$ ). Temperature variations were not regarded as significant challenges to microbial colonization

12 compared to other variables (Supplementary Material, Fig. S6). The paleo-playa site 10 failed to

13 yield recoverable DNA from any depth, thus highlighting that ancient landforms created under

14 moisture sufficiency, and with elevated anhydrite levels as indicated by the rover's Raman

15 spectrometer (Supplementary Material, Fig. S5) may not support extant genetic biosignatures and

16 do not support recoverable relic DNA. Our overall DNA recovery success was consistent with our

17 expectations for the driest desert location on Earth. It is notable that we have observed Antarctic

18 mineral sediments, another Mars analogue, generally yield higher recovery rates using similar

19 methodologies, e.g. (Lee et al., 2012; Pointing et al., 2009). However, this is due to enhanced

20 standing biomass due to the less extreme nature of the growing season in Antarctic desert where

21 long periods of frozen hibernation are punctuated by periods of moisture sufficiency during the

22 austral summer (Pointing et al., 2015).

Clear patterns were discernible from manually excavated sediment pits. Bacterial diversity

24 decreased significantly with depth across both terrains (Chao1 richness: $\mathrm{r}=0.508, \mathrm{p}=0.044$;

25 Shannon's H: $\mathrm{r}=0.731, \mathrm{p}=0.001)$, thus indicating depth as a major driver of bacterial diversity 
1 (Supplementary Material, Fig. S8, Table S6). Bacterial communities formed six diversity clusters

2 that associated with clearly defined depth ranges and zones of variability in sediment moisture

3 and geochemistry (Fig. 2a). Subsurface communities were more distinct between habitat types but

4 also more heterogenous overall than surface-associated communities, due largely to the lower

5 bacterial diversity within each depth-defined sediment micro-habitat. Community evenness

6 displayed little clear pattern with depth for desert pavement but for playa deep samples displayed

7 very low evenness reflecting their highly specialized diversity (Supplementary Material, Table

8 S6). In all cases the rover-acquired samples showed a generally similar pattern in depth profile

9 and diversity clusters although these were less clearly defined and we attributed this to mixing of

10 sediment during recovery using the bite-drilling approach (Fig. 2b; Supplementary Material, Fig.

11 S8).

To further unravel the influence of sediment environment on bacterial diversity, we

13 performed canonical correspondence analysis (CCA) to establish the association of distinct

14 geochemistry for desert pavement and playa samples on the assembly of bacterial communities

15 (Fig. 1). Surface communities were strongly influenced by P levels that were elevated compared

16 to the subsurface (Fig. 1). Bacterial diversity in subsurface sediment habitats correlated with two

17 groups of geochemical variables: The playa subsurface community was strongly associated with

18 EC and extractable cations $\left(\mathrm{Ca}^{2+}, \mathrm{Mg}^{2+}, \mathrm{Na}^{+}\right)$and the rover-mounted Raman data indicated

19 elevated anhydrite and gypsum (Supplementary Material, Table S3, Fig. S5). This strongly

20 suggests that bio-availability of water may be limited in this subsurface habitat despite relatively

21 high moisture levels due to the effects of salt saturation. Conversely the pavement

22 subsurface community was associated largely with $\mathrm{pH}$, and also $\mathrm{C}$ and $\mathrm{K}^{+}$although the magnitude

23 of variation for these variables was low. This $\mathrm{pH}$-dependent structuring of microbial diversity is

24 consistent with observations for global trends in soil microbial diversity (Fierer and Jackson,

25 2006). The BEST multiple rank correlation routine was employed to further examine our data and 
1 rank the relative association of abiotic variables with the observed bacterial diversity as follows:

2 extractable cations $\left(\mathrm{Ca}^{2+}, \mathrm{Mg}^{2+}, \mathrm{Na}^{+}\right)$and phosphorous $>$sulfate-sulfur $>>\mathrm{EC}(p w=0.595-0.609, p$

$3<0.05)$, thus further validating the ordinations and CCA analysis.

$5 \quad$ Highly specialized sediment bacterial communities

6 The taxa identified in sediment indicated highly specialized and relatively low diversity bacterial

7 communities in both desert pavement and playa, and this low diversity reflects global trends in

8 soil microbial diversity where deserts are considered to be relatively depauperate (Delgado-

9 Baquerizo et al., 2018; Thompson et al., 2017). Overall the drill samples yielded weaker depth

10 resolution than manual sampling but still corroborated observations from the manually collected

11 samples (Fig. 3, Supplementary Material Fig. S9). Bacterial taxonomic diversity varied

12 significantly with subsurface habitat as compared to a generally consistent surface community

13 (Fig. 3) (Crits-Christoph et al., 2013). Overall, communities were dominated largely by only three

14 phyla: Chloroflexi, Actinobacteria and Alphaproteobacteria.

All surface communities were dominated by the AKIW781 lineage of the

16 photoheterotrophic Chloroflexi, a known desert soil crust taxon (NCBI GenBank accession

17 number: JQ402146), and overall community structure contributed additional taxonomic resolution

18 to previous observations of surface sediment communities (Crits-Christoph et al., 2013; Schulze-

19 Makuch et al., 2018). AKIW781 has also been recorded in desert varnish on rock surfaces

20 (Kuhlman et al., 2008) as well as a keystone taxon of hypolithic communities (Lacap et al., 2011)

21 in the Atacama. This indicates a cosmopolitan distribution and broad habitat preference among

22 surface niches in this extreme desert. Conversely, the Chloroflexi were minor components of

23 subsurface communities, decreasing in relative abundance with depth, and mainly comprised an

24 uncharacterized candidate class Ellin6529, likely adapted to non-phototrophic metabolism in the

25 subsurface microhabitat. 
Low and mid-depth subsurface horizons for both desert pavement and playa were

dominated by the low G-C gram positive Actinobacteria (Fig. 3, Supplementary Material, Fig.

3 S9). They are typically thick-walled bacteria commonly encountered in soil. Communities

4 encountered were specific to each depth horizon and shifts in diversity clearly reflected the

5 moisture zones identified for both pavement and playa horizons. At shallow depths $(<200 \mathrm{~mm})$

6 halotolerant, alkalotolerant, spore-forming desiccation-tolerant actinobacterial groups were

7 abundant and included the orders acidimicrobiales, gaiellales, nitriliruptorales and

8 solirubrobactales in desert pavement and acidimicrobiales and nitriliruptorales in playa. Mid-

9 depth sediments $(300-<500 \mathrm{~mm})$ corresponding to the zone of greatest moisture availability in

10 both substrates supported highly variable bacterial diversity between samples and also the most

11 diverse communities (Supplementary Material, Table S6). Of note was the elevated relative

12 abundance of bacillales at a single pavement site and this may reflect the relatively high altitude

13 and limited moisture input to the subsurface. The bacillales-dominated community was more

14 similar to those encountered at greater depths at other locations. The bacillales are characterized

15 by their ability to form highly resistant endospores and so may indicate an increasingly

16 challenging micro-habitat in deeper subsurface sediments.

There were fewer deep sediment samples from which to make comparisons and this

18 reflected the more challenging subsurface environment, and deeper communities $(500+\mathrm{mm})$

19 generally displayed lowest taxonomic diversity. The deepest desert pavement community was

20 similar to those at shallower depths but displayed elevated relative abundance of acidimicrobiales.

21 Conversely the deep playa community shifted to a fundamentally different composition from

22 shallower depths that was dominated almost exclusively by a single facultative methylotrophic and desiccation-tolerant Methylobacterium radiotolerans taxon (NCBI GenBank accession

24 number: LT985974) from the phylum Alphaproteobacteria. We speculate the C1 metabolism of 25 this taxon allows it to exploit simple $\mathrm{C} 1$ compounds as well as subsurface methane sources (Kao 
et al., 2017), a molecule known to be released from subsurface sources on Mars (Stevens et al., 2017). An overall picture thus emerges of highly-specialized bacterial diversity adapted to and reflecting the challenging subsurface habitat as well as reflecting moisture availability zones determined at least in part by sediment depth and geochemistry.

Other bacteria typically encountered in desert surface communities and generally regarded as tolerant to extreme conditions were not major components of subsurface communities. A complete absence of cyanobacterial taxa was consistent with their adaptation to surface mineral refugia rather than subsurface sediment habitats that preclude photoautotrophy (Azua-Bustos et al., 2012), and the highly desiccation-tolerant Deinococcus-Thermus group were represented by only a single lineage of Trueperaceae candidate genus B-42 recovered in just a few subsurface samples with low abundance $(0.6-4.8 \%)$, compared to a relatively diverse assemblage of cultivable Deinococci recovered from surface sediments in other less arid desert locations (Rainey et al., 2005). This further supports our evidence for highly specialized subsurface communities selected for by the distinct geochemistry and microclimate in the subsurface Mars analogue sediments of the Atacama. They may thus be broadly indicative of the type of microbial consortia that could exploit Martian subsurface habitats.

In the deepest and least-diverse playa communities biocomplexity was reduced almost to a single taxon, reflecting extreme selective pressure and also highlighting a possible lack of resilience to environmental change given that recruitment to deep subsurface sediments may be limited. The minimum biocomplexity may comprise multiple ecotypes of a single taxon, each adapted to exploit a given suite of microclimate and geochemical conditions (Koeppel et al., 2008). They exhibited a strong preference for $\mathrm{C} 1$ and/or autotrophic taxa that are somewhat delinked from their immediate surroundings in terms of carbon sequestration and reflect the extreme oligotrophic nature of these microhabitats. Given the highly specialized diversity recovered, the association of putative physiology with environmental variables, and lack of DNA recovery in 
1 paleo-playa samples; we conclude that our estimates are likely to represent resident microbial

2 communities rather than relic DNA (Carini et al., 2016; Schulze-Makuch et al., 2018).

\section{Implications for detection of biosignatures on Mars}

5 The autonomous rover drilling platform yielded subsurface sediment samples that allowed for the

6 first time a combined investigation of sediment abiotic properties and microbial diversity at an

7 unprecedented level of detail. The parallel manual sampling and analysis demonstrated feasibility

8 and fidelity of the autonomous rover approach. Highly specialized but low-diversity subsurface

9 bacterial communities were encountered patchily and this was strongly associated with abiotic

10 variables which suggests that "follow the water" is only part of the biosignature exploration

11 solution in the search for potential habitable refuges on Mars. Consideration of subsurface micro-

12 habitat variability in geochemistry, originating with and adapted to possible water availability

13 zones may also be key. Whilst the geochemistry of our analogue sites was similar to that of a

14 habitable Martian regolith, moisture in the Atacama is surface-sourced by fog and/or rain events

15 (McKay et al., 2003), whereas on Mars subsurface sources may provide an upward migration of

16 moisture similar to that observed in Antarctic mineral sediment overlaying permafrost (Goordial

17 et al., 2016; Stomeo et al., 2012). Thus, extrapolating habitable subsurface locations on Mars

18 would need to consider this along with the incident radiation regime and other Martian

19 environmental variables. Based on the exploratory design consideration for this NASA-funded

20 research, drill depth was constrained at $800 \mathrm{~mm}$ as a proof of concept study. However, current

21 plans are for both NASA and ESA to target depths of up to $2 \mathrm{~m}$ on Mars in order to target

22 potential subsurface habitats that account for Martian environmental variables.

The relevance of ecology and microbial habitats to past and possible extant life on Mars

24 are finally coming to the fore in the robotic search for biosignatures on Mars (Cabrol, 2018;

25 Warren-Rhodes et al., 2007). As our study suggests, detecting such life or its residual 
biosignatures may prove highly challenging, given that in the most extreme deserts on Earth these communities are extremely patchy in distribution and occur with exceedingly low biomass. The drill apparatus employed in this study has demonstrated that subsurface sediment biosignatures can be autonomously recovered, although precise depth delineation requires refinement with the bite-drilling approach used in this study. Whilst genetic biosignatures such as DNA used in our study may not ultimately be the primary method employed to search for traces of life on Mars, they are the most reliable and widely used method currently available for microbial diversity estimation (Delgado-Baquerizo et al., 2018; Thompson et al., 2017). This approach provided essential first proof of concept that an incontrovertible biological signature within the likely range for geochemical variables in a habitable subsurface environment can be recovered from a Marslike sediment using an autonomous rover.

\section{Acknowledgements}

The work was completed with funding from NASA Astrobiology Science and Technology for Exploring Planets (ASTEP) Program Grant NNX11AJ87G and a Yale-NUS College Start-Up Grant. NASA Life in the Atacama Project team members that contributed to the success of this research: Guillermo Chong (Universidad Católica del Norte), Cecilia Demargasso (Universidad Católica del Norte), Greydon Foil (Carnegie-Mellon University), Christopher Gayle Tate (University of Tenessee), Trent Hare (US Geological Survey), Donnabella C. Lacap-Bugler (Auckland University of Technology), Jeff Moersch (University of Tenessee), Ken Tanaka (US Geological Survey), Cinthya Tebes (Universidad Católica del Norte), Srinivasan Vijayarangan (Honeybee Robotics and Spacecraft Mechanisms Corp.), Michael Wagner (Carnegie-Mellon University), Alian Wang (Washington University), Jie Wei (Washington University). The authors are grateful to Brad Sutter (NASA) for advice on interpretation of geochemical analysis, and Craig Cary (University of Waikato) for assistance with biosecurity compliance and sample 
1 custody. Author Kris Zacny was employed by Honeybee Robotics and Spacecraft Corporation.

2 All other authors declare no competing interests. A preprint version of this manuscript is available

3 on the bioRxiv server: https://www.biorxiv.org/content/early/2018/06/20/269605.

\section{$5 \quad$ References}

6 Azua-Bustos, A., Urrejola, C., and Vicuña, R. (2012). Life at the dry edge: Microorganisms of the

Bagaley, D. R. (2006). Uncovering bacterial diversity on and below the surface of a hyper-arid environment, The Atacama Desert, Chile. PhD Thesis, Louisiana State University.

Cabrol, N. A. (2018). The Coevolution of Life and Environment on Mars: An Ecosystem Perspective on the Robotic Exploration of Biosignatures. Astrobiology 18, 1-27. doi:10.1089/ast.2017.1756.

Carini, P., Marsden, P. J., Leff, J. W., Morgan, E. E., Strickland, M. S., and Fierer, N. (2016). Relic DNA is abundant in soil and obscures estimates of soil microbial diversity. Nat. Microbiol. 2, 16242. doi:10.1038/nmicrobiol.2016.242.

Clarke, J. D. A. (2006). Antiquity of aridity in the Chilean Atacama Desert. Geomorphology 73, 101-114. doi:10.1016/j.geomorph.2005.06.008.

Connon, S. S. A., Lester, E. D. E., Shafaat, H. H. S., Obenhuber, D. C., and Ponce, A. (2007). Bacterial diversity in hyperarid Atacama Desert soils. J Geophys Res - Biogeosci 112, G04S17. doi:10.1029/2006JG000311.

Crits-Christoph, A., Robinson, C. K., Barnum, T., Fricke, W. F., Davila, A. F., Jedynak, B., et al. (2013). Colonization patterns of soil microbial communities in the Atacama Desert. Microbiome 1, 28. doi:10.1186/2049-2618-1-28.

Delgado-Baquerizo, M., Oliverio, A. M., Brewer, T. E., Benavent-González, A., Eldridge, D. J., Bardgett, R. D., et al. (2018). A global atlas of the dominant bacteria found in soil. Science 
(80-. ). 359, 320-325. doi:10.1126/science.aap9516.

Drees, K. P., Neilson, J. W., Betancourt, J. L., Quade, J., Henderson, D. A., Pryor, B. M., et al. (2006). Bacterial community structure in the hyperarid core of the Atacama Desert, Chile. Appl Env. Microbiol 72, 7902-7908.

Ewing, S. A., Sutter, B., Owen, J., Nishiizumi, K., Sharp, W., Cliff, S. S., et al. (2006). A threshold in soil formation at Earth's arid-hyperarid transition. Geochim. Cosmochim. Acta 70, 5293-5322. doi:10.1016/j.gca.2006.08.020.

Farley, K., and Williford, K. (2017). Seeking Signs of Life and More: NASA's Mars 2020 Mission. EOS Trans. - Am. Geophys. Union 98, doi: 10.1029/2017EO066153. doi:10.1029/2017EO066153.

Fierer, N., and Jackson, R. B. (2006). The diversity and biogeography of soil bacterial communities. Proc. Natl. Acad. Sci. U S A 103, 626-631. doi:10.1073/pnas.0507535103.

Goordial, J., Davila, A., Lacelle, D., Pollard, W., Marinova, M. M., Greer, C. W., et al. (2016). Nearing the cold-arid limits of microbial life in permafrost of an upper dry valley, Antarctica. ISME J. 10, 1613-1624. doi:10.1038/ismej.2015.239.

Hartley, A. J., Chong, G., Houston, J., and Mather, A. E. (2005). 150 million years of climatic stability: evidence from the Atacama Desert, northern Chile. J. Geol. Soc. London. 162, 421424. doi:10.1144/0016-764904-071.

Kao, Y., Li, J., Wang, Y., Li, C., Yu, B., Yao, M., et al. (2017). Scale-dependent key drivers controlling methane oxidation potential in Chinese grassland soils. Soil Biol. Biochem. 111, 104-114. doi:10.1016/J.SOILBIO.2017.04.005.

Koeppel, A., Perry, E. B., Sikorski, J., Krizanc, D., Warner, A., Ward, D. M., et al. (2008). Identifying the fundamental units of bacterial diversity: a paradigm shift to incorporate ecology into bacterial systematics. Proc. Natl. Acad. Sci. U. S. A. 105, 2504-9. doi:10.1073/pnas.0712205105. 
1 Kuhlman, K. R., Venkat, P., La Duc, M. T., Kuhlman, G. M., and McKay, C. P. (2008). Evidence of a microbial community associated with rock varnish at Yungay, Atacama Desert, Chile. $J$. Geophys. Res. 113, G04022. doi:10.1029/2007JG000677.

Lacap, D. C., Warren-Rhodes, K. A., McKay, C. P., and Pointing, S. B. (2011). Cyanobacteria and chloroflexi-dominated hypolithic colonization of quartz at the hyper-arid core of the Atacama Desert, Chile. Extremophiles 15, 31-8. doi:10.1007/s00792-010-0334-3.

Lee, C. K., Barbier, B. A., Bottos, E. M., McDonald, I. R., Cary, S. C., and Barbier, B. A. (2012). The Inter-Valley Soil Comparative Survey: the ecology of Dry Valley edaphic microbial communities. ISME J 6, 1046-1057. doi:10.1038/ismej.2011.170.

Lester, E. D., Satomi, M., and Ponce, A. (2007). Microflora of extreme arid Atacama Desert soils. Soil Biol. Biochem. 39, 704-708. doi:10.1016/j.soilbio.2006.09.020.

Martin-Torres, F. J., Zorzano, M.-P., Valentin-Serrano, P., Harri, A.-M., Genzer, M., Kemppinen, O., et al. (2015). Transient liquid water and water activity at Gale crater on Mars. Nat. Geosci 8, 357-361. doi:10.1038/ngeo2412.

McKay, C. P. (2014). Requirements and limits for life in the context of exoplanets. Proc. Natl. Acad. Sci. U. S. A. 111, 12628-33. doi:10.1073/pnas.1304212111.

McKay, C. P., Friedmann, E. I., Gomez-Silva, B., Caceres-Villanueva, L., Andersen, D. T., Landheim, R., et al. (2003). Temperature and Moisture Conditions for Life in the Extreme Arid Region of the Atacama Desert: Four Years of Observations Including the El Nino of 1997-1998. Astrobiology 3, 393-406. doi:10.1089/153110703769016460.

Navarro-Gonzalez, R., Rainey, F. A., Molina, P., Bagaley, D. R., Hollen, B. J., de la Rosa, J., et al. (2003). Mars-like soils in the Atacama Desert, Chile, and the dry limit of microbial life. Science. 302, 1018-1021.

Peel, M. C., and Finlayson, B. L. (2007). Updated world map of the Köppen-Geiger climate classification. Hydrol. Earth Syst. Sci. 11, 1633-1644. doi:10.5194/hess-11-1633-2007. 
1 Pointing, S. B., and Belnap, J. (2012). Microbial colonization and controls in dryland systems. Nat. Rev. Microbiol. 10, 551-562. doi:10.1038/nrmicro2831.

3 Pointing, S. B., Buedel, B., Convey, P., Gillman, L. N., Koerner, C., Leuzinger, S., et al. (2015).

Biogeography of photoautotrophs in the high polar biome. Front. Plant Sci. Funct. Plant Ecol. 6, 692. doi:10.3389/fpls.2015.00692.

Pointing, S. B., Chan, Y., Lacap, D. C., Lau, M. C. Y., Jurgens, J. A., and Farrell, R. L. (2009). Highly specialized microbial diversity in hyper-arid polar desert. Proc. Natl. Acad. Sci. U. S. A. 106, 19964-19969. doi:10.1073/pnas.0908274106.

Rainey, F. A., Ray, K., Ferreira, M., Gatz, B. Z., Nobre, M. F., Bagaley, D., et al. (2005). Extensive diversity of ionizing-radiation-resistant bacteria recovered from Sonoran Desert soil and description of nine new species of the genus Deinococcus obtained from a single soil sample. Appl. Environ. Microbiol. 71, 5225-5235.

Schulze-Makuch, D., Wagner, D., Kounaves, S. P., Mangelsdorf, K., Devine, K. G., de Vera, J.P., et al. (2018). Transitory microbial habitat in the hyperarid Atacama Desert. Proc. Natl. Acad. Sci. U. S. A. 115, 2670-2675. doi:10.1073/pnas.1714341115.

Stevens, A. H., Patel, M. R., and Lewis, S. R. (2017). Modelled isotopic fractionation and transient diffusive release of methane from potential subsurface sources on Mars. Icarus 281, 240-247. doi:10.1016/j.icarus.2016.08.023.

Stomeo, F., Makhalanyane, T. P., Valverde, A., Pointing, S. B., Stevens, M. I., Cary, C. S., et al. (2012). Abiotic factors influence microbial diversity in permanently cold soil horizons of a maritime-associated Antarctic Dry Valley. FEMS Microbiol Ecol 82, 326-340. doi:10.1111/j.1574-6941.2012.01360.x.

Thompson, L. R., Sanders, J. G., McDonald, D., Amir, A., Ladau, J., Locey, K. J., et al. (2017). A communal catalogue reveals Earth's multiscale microbial diversity. Nature 551, 457-463. doi:10.1038/nature24621. 
1 Vago, J. L., Westall, F., Pasteur Instrument Teams, Landing S, A. J., Coates, A. J., Jaumann, R., Korablev, O., et al. (2017). Habitability on Early Mars and the Search for Biosignatures with the ExoMars Rover. Astrobiology 17, 471-510. doi:10.1089/ast.2016.1533.

Warren-Rhodes, K., Lee, K., Archer, S., Lacap, D., Ng-Boyle, L., Wettergreen, D., et al. (2018). Subsurface microbial habitats in an extreme desert Mars-analogue environment. bioRxiv, 269605. doi:10.1101/269605.

Warren-Rhodes, K., Weinstein, S., Piatek, J. L., Dohm, J., Hock, A., Minkley, E., et al. (2007). Robotic ecological mapping: Habitats and the search for life in the Atacama Desert. $J$. Geophys. Res. Biogeosciences 112. doi:10.1029/2006JG000301.

Wei, J., Wang, A., Lambert, J. L., Wettergreen, D., Cabrol, N., Warren-Rhodes, K., et al. (2015). Autonomous soil analysis by the Mars Micro-beam Raman Spectrometer (MMRS) on-board a rover in the Atacama Desert: a terrestrial test for planetary exploration. J. Raman Spectrosc. 46, 810-821. doi:10.1002/jrs.4656.

Zacny, K., Zacny, G., Paulsen, S., Yoon, D., Wettergreen, D., and Cabrol, N. (2014). Life in the Atacama - The drill and sample delivery system. Results from the 2013 field campaign. in Proceedings of the 45th Lunar and Planetary Science Conference, 1174. 


\section{$1 \quad$ Figure legends}

2 Figure 1. Correlation of Mars analogue sediment geochemistry with bacterial diversity.

3 Canonical Correspondence Analysis (CCA) triplot with symmetrical scaling indicating

4 differences in sediment geochemistry within sediment pits, and influence of these abiotic

5 variables on bacterial communities and individual taxa. The three most abundant taxa are labelled

6 (A, B, C). The circle size of each sample indicates species richness index (Chao 1) of the

7 respective community.

9 Figure 2. Depth-defined bacterial diversity in Mars analogue sediments of the Atacama

10 Desert. Non-metric multidimensional scaling (NMDS) ordination of Bray Curtis similarities for

11 bacterial diversity versus sediment depth from a) manual recovery, and b) rover recovery. Shaded

12 areas indicate similarity clusters for sediment communities at the same depth. The size of each

13 symbol (circle or triangle) indicates species richness index (Chao 1) of the respective community.

14 Mid-range values were used for drill samples where depth ranges instead of individual depths

15 were generated.

16

17 Figure 3. Highly specialized bacterial communities in Mars analogue sediment depth

18 horizons. Distribution of bacterial diversity by taxonomic class with sediment depth for manual

19 (M) and autonomous rover drill (D) recovered samples. Colored shading indicates relative

20 abundance within each community for a given bacterial class. Grey shading indicates no

21 recoverable bacteria. 


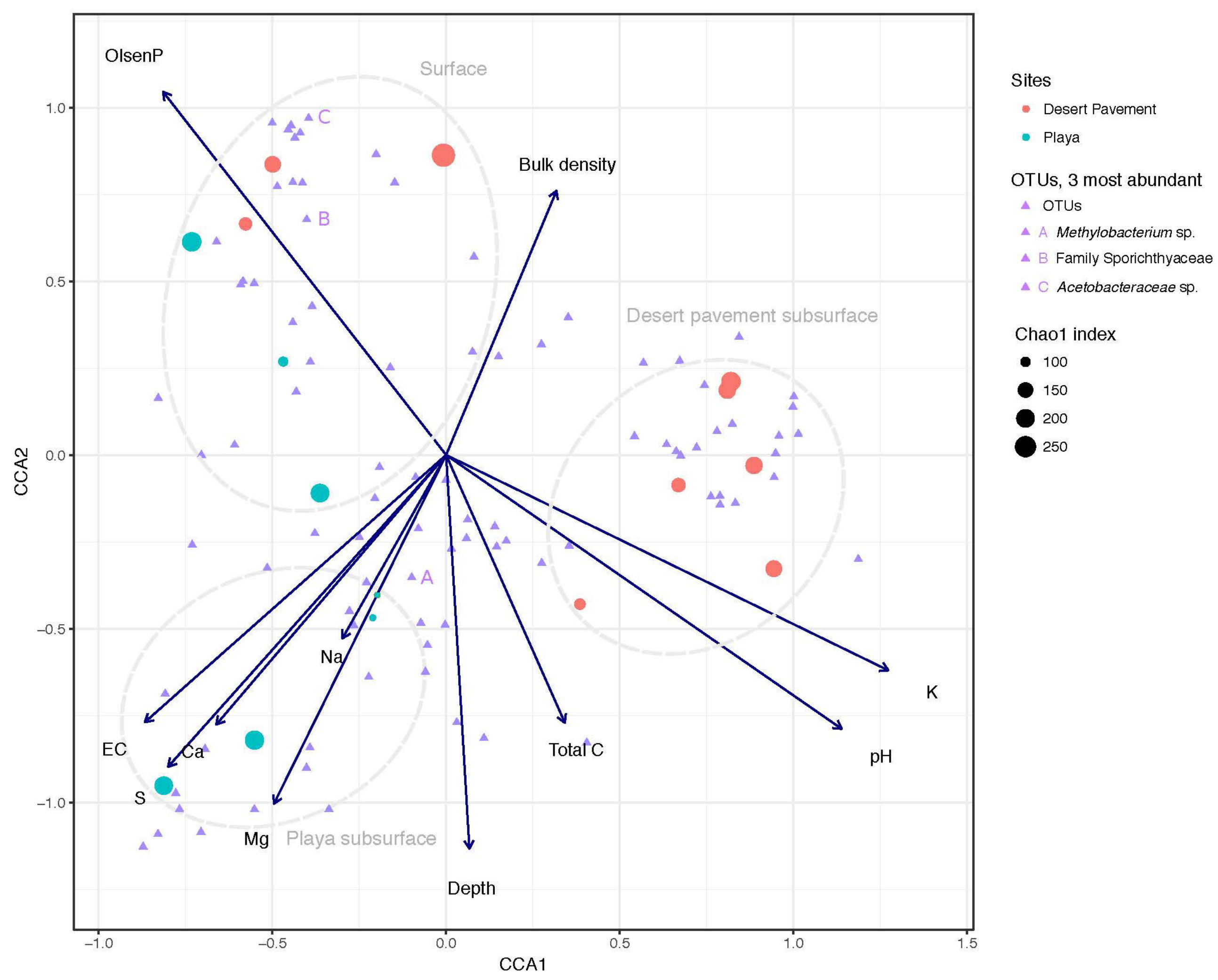




\section{a}

递

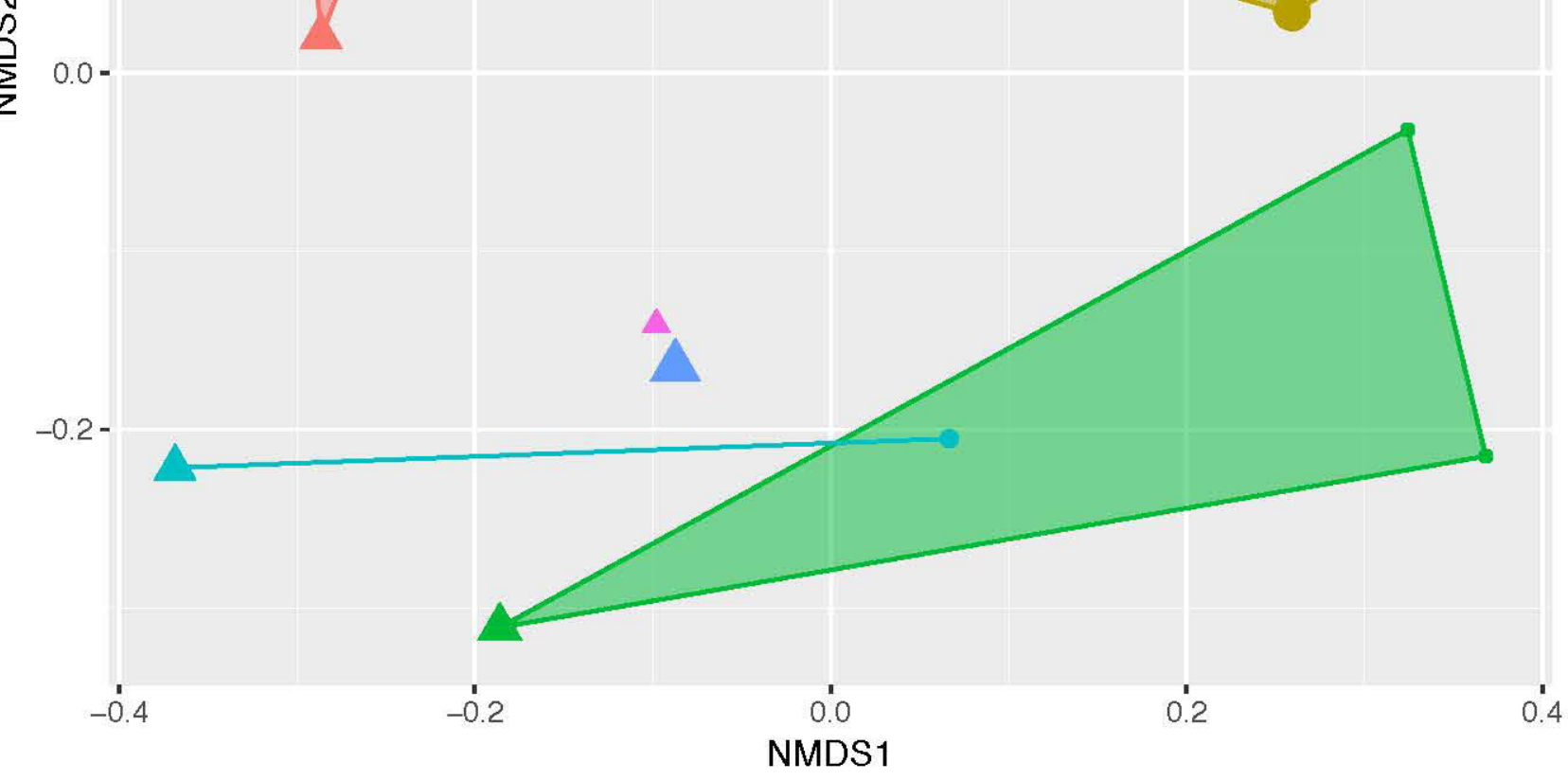

\section{Sites}

- Desert Pavement

- Playa

Chao1 index

- 100

- 150

200

250

\section{b}

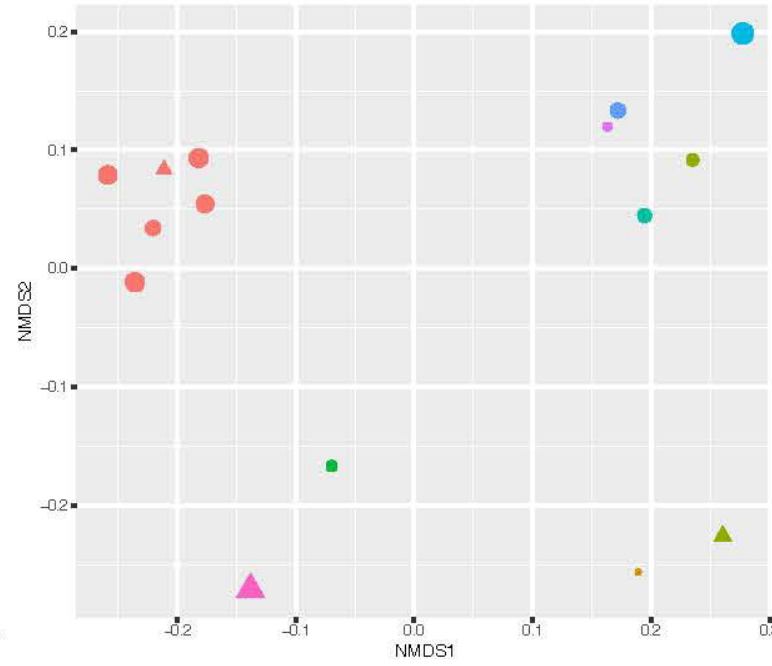

Sample depth $(\mathrm{mm})$

\begin{tabular}{|l|l|}
\hline - & 0 \\
\hline \hline & 100 \\
\hline - & 200 \\
\hline - & 300 \\
\hline - & 500 \\
\hline - & 700 \\
\hline
\end{tabular}

Sample depth (mm)

$\because 100$

150
$: 300$

- 30

- 385

. 630

.700

Chao1 index

- 100

$:^{200}$

300 
